\title{
Effect of the First Coagulating Bath \\ Composing on the Structure of PES Membrane
}

\author{
Zhenlei Li \\ Key Lab of Hollow Fiber Membrane Materials \& Membrane Process, Tianjin Polytechnic University \\ Tianjin 300160, China
}

Tel: 86-22-24528205_E-mail: leily1116@msn.com

\begin{abstract}
Make PES flat-sheet style membrane through the method of dual-bath coagulation method. Change the composing of the first coagulating bath and make the casting membrane liquid stay in the first coagulating bath for enough time and control the surface and interior structure of the membrane at the same time. Study the interior and exterior structure when the DMAc mass fraction of first coagulating bath is among 0\%-70\%, and find out with the increase of mass fraction, the interior structure of membrane transits from the form of finger pore to the form of spongy pore, and when the mass fraction of the first coagulating bath achieves $70 \%$, there is no structure with the form of finger pore, but the opening structure on the membrane surface appears when the mass fraction is $60 \%$ and increases with the increase of concentration. When the mass fraction of the first coagulating bath is among $0 \%-50 \%$, the pure water flux of the membrane decreases with the concentration, and the rejection ratio of BSA change less, and when the concentration of the first coagulating bath exceeds $60 \%$ and the openings appear on the membrane surface, the pure water flux would ascend but the rejection ratio would markedly decrease.
\end{abstract}

Keywords: Dual-bath coagulation method, Flat-sheet style membrane, Surface opening

\section{Introduction}

At the beginning of 1960 s, Loeb and his colleagues developed the method which used the phase inversion method to make asymmetrical separation membrane. Hence, the phase inversion method became one of main methods making asymmetrical membrane (Wei, 2005). The traditional phase inversion method influences dual-diffusion speeds through changing the temperature of coagulating bath to achieve the purpose of controlling the membrane structure. In 1991, Lin Yizheng put forward the method that adopted dual-bath coagulation method to make asymmetrical separation membrane and the casting membrane liquid was immerged into different coagulation baths in turn, and achieved the purpose of controlling the pore structure of membrane surface through dealing with the membrane surface by changing the composing of the first coagulation bath (Lin, 1991). This method can effectively controls the surface structure of the membrane, but if the stay time of casting membrane liquid in the first coagulation bath is increased, the membrane surface and interior structure can be the controlled through changing the composing of the first coagulation bath, accordingly the more plentiful membrane infrastructural materials can be offered for the mixed membrane and composite membrane.

This article adopts the dual-bath coagulation method, takes the DMAc liquor as the first coagulation bath and ultrafiltration water as the second coagulation bath, prolongs the stay time of the casting membrane liquid in the first coagulation bath, and studies the effect of the first coagulation bath mass fraction on the membrane surface and interior structure and changing situations of membrane pure water flux and BSA rejection ratio.

\section{Experiment}

\subsection{Raw materials and reagents}

The raw materials are membrane-based PES and BASF. The analytical reagents include N, N-NMP, DMAc, pure ethanol, glycerol and acetone, which are produced by Tianjin Fengchuan Chemical Reagent Technology Co., Ltd.

\subsection{Process of membrane making}

In the general membrane making process with dual-bath coagulation method, the stay time of casting membrane liquid in the first coagulation bath is very short, and the main function of the first coagulation bath is to preliminarily adjust the polymer or impregnant content on the membrane surface, and the membrane is fully formed by the second 
coagulation bath (Zhu, 2005, p.17-19). Though the structure of the membrane surface can influence the dual-diffusion speeds to some extent and accordingly influence the interior structure of the membrane, but because the membrane surface is very thin and has few influences to the dual-diffusion, so the interior structure of the membrane is mainly effected by the characters of the second coagulation bath (Zhao, 2005, p.93-96). This experiment increases the stay time of membrane in the first coagulation, and make the interior of the membrane influenced by the first coagulation, and change the interior structure of the membrane through by the first coagulation bath.

The membrane making process is basically same to the common flow, which dissolves some certain PES in the DMAc, and adds PEG, then intensely mixes round to equal and stable polymer liquor. Take the liquor pressurized in $50{ }^{\circ} \mathrm{C}$ and placed to mix for 24 hours. Use the metal stick to quickly scratch the casting membrane liquid on the glass board to make the membrane, then put the glass board into the first coagulation bath and stay for enough time to make the first coagulation bath and materials in the membrane liquid fully exchanged, finally put the glass board into the water bath. Because the first coagulation DMAc mass fraction adopted in this experiment is between 0\%-70\%, quite a few parts of membranes have been coagulated in the first coagulation bath. When the concentration of the first coagulation DMAc exceeds $50 \%$, the membranes can not be fully formed in the first coagulation bath.

\subsection{Performances and tokens of the membrane}

\subsubsection{Pure water flux mensuration of the membrane}

We adopt the ultrafiltration evaluation device made by Tianjin Motimo Group to measure the flux. Select one piece of membrane without surface bugs and place it on the flat poor, press it, adjust the pressure to $0.1 \mathrm{MPa}$ and maintain the water temperature in $25^{\circ} \mathrm{C}$, and when the system becomes stable, measure the flux of the membrane. Compute the water flux according to the following formula.

$Q=\frac{V}{A t}$

Where, Q represents the water flux $\left(\mathrm{L} / \mathrm{m}^{2} \cdot \mathrm{h}\right), \mathrm{V}$ represents the volume of the permeated liquid $(\mathrm{L})$, A represents the effective acreage of the membrane $\left(\mathrm{m}^{2}\right)$ and $\mathrm{t}$ represents the ultrafiltration time (s).

\subsubsection{Rejection ratio mensuration of the membrane}

Select BSA with 67 thousand molecular weight as the solute, and select cushion liquor of $\mathrm{PH}=7.4$ to confect BSA liquor of $1 \mathrm{~g} / \mathrm{l}$, and maintain the liquor temperature of $20{ }^{\circ} \mathrm{C}$, filtrate that liquor by the PES membrane made, take out the original liquor and the corresponding sieved liquor, then measure their absorbencies by UV2450 ultraviolet spectrometer. The computation formula of rejection ratio is $R=\frac{E_{o}-E_{S}}{E_{o}} \times 100 \%$.

Where, $\mathrm{R}$ represents the rejection ratio, $\mathrm{E}_{\mathrm{o}}$ represents the absorbency of the original liquor and $\mathrm{E}_{\mathrm{s}}$ represents the absorbency of the sieved liquor.

\section{Experiment results and discussions}

\subsection{Effect of the first coagulation bath on the membrane structure}

From Figure 1, we can see that with the increase of the content of DMAc in the coagulation bath, the interior of the PES membrane transits from the form of finger pore to the form of spongy pore. When the mass fraction of the coagulation bath achieves $70 \%$ (see A3 in Figure 1), the opening structure on the surface of the PES membrane appears, which aperture is about among 0.1-2 $\mu \mathrm{m}$ enough passing polymer of high molecule.

For the cross section structure of the membrane (see B1, B2 and B3 in Figure 1), with the increase of mass fraction of the coagulation bath, the long and big finger pores gradually becomes short and small and the spongy pores gradually becomes more and more, finally the sponge pore structure is formed. The coagulation bath with $30 \%$ of DMAc (see B1 in Figure 1) forms the classic finger pore structure, and the coagulation bath with $70 \%$ of DMAc (see B3 in Figure 1) forms the multi-pore sponge structure. Because the membrane fully immerges in the first coagulation bath, the interior and exterior of the membrane are preliminarily coagulated in the first coagulation bath. In A1 of Figure 1, because of much water contents, fewer DMAc contents, quick dual-diffusion speed, high concentration grads of nonsolvent water between the interior and exterior of the casting membrane liquor, and quicker diffusion speed than the solvent, much nonsolvent makes the parts of poor phase in the casting membrane liquor grow up quickly and form the finger pore structure. With the increase of DMAc in the coagulation bath, the concentration grads of the water decrease, the diffusion speed decreases, the separation speed slows down, and the finger pores become denser and smaller. The DMAc contents of the first coagulation bath in A3 and B3 of Figure 1 are very high and achieve the contents of casting membrane liquor, and the water contents are very low, which can make the casting membrane liquor only separate but not coagulate, and forms the disperse poor phase and rich phase structure of the polymer, and after entering the second coagulation bath, the rich phase coagulates to form membrane and the poor phase form multi-pore structure. Because of equal decentralization of the poor phase and rich phase in the membrane, so the opening structure also appears on the surface layer of the membrane. 


\subsection{Effect of coagulation bath composing on pure water flux of the membrane}

The common membrane is composed of dense function layer on the surface and the under support layer. Usually the function layer is thin and the support layer is thick. The interior multi-pore support layer mainly influences the membrane water flux, and the pores in the support layer are smaller and less, the flux is lower. At the same time, when the big pore structure appears in the surface function layer of the membrane, the water flux will increase (Li, 1994, p.329-340).

In Figure 2, with the increase of DMAc mass fraction in the coagulation bath, the pure water flux presents the tendency descending first and ascending then. When the DMAc mass fraction is among $0 \%-50 \%$, the pure water flux of PES membrane descends. When the DMAc mass fraction is in 50\%, the pure water flux of PES membrane achieves the minimal value. When the DMAc mass fraction is among 50\%-70\%, the pure water flux of PES membrane begins to ascend.

The water content of the coagulation bath is the main factor to influence the microstructure that the casting membrane liquor coagulates to the membrane. When the water content is high, in the instant that the casting membrane liquor contact with the coagulation bath, the dual-diffusion is very intense, and the surface layer with dense structure on the surface of the casting membrane liquor, and the interior forms bigger finger pore structure, and when the water content is low, the dualdiffusion and membrane forming speed are slow, the surface layer of the membrane is thick, and the interior of the membrane forms spongy pore structure (Sun, 2003, p.102-105). Therefore, when the DMAc mass fraction of the coagulation bath is among $0 \%-50 \%$, with the increase of DMAc concentration, the structure of the support layer of the membrane transits from finger pore to spongy pore, and the finger pore becomes smaller and smaller, the surface layer of the membrane becomes thick with it, and the pure water flux descends (He, 2003, 2151-2157). When the DMAc concentration exceeds 50\%, the separation process not only becomes slow, but can not complete separation in the first coagulation bath because of too high DMAc contents, and the separation of rich phase and poor phase appears, the solidifying process would be completed in the second coagulation bath. Here, the opening structure appears on the formed surface of the membrane, and the interior of the membrane is multi-pore spongy pores, and because of existence of surface opening, the function layer almost has no effect on the water flux. So the pure flux ascends again when the DMAc concentration exceeds $50 \%$.

\subsection{Effect of coagulation bath composing on BSA rejection ratio of the membrane}

The rejection ability of the membrane is mainly embodied by the function layer of the membrane. The pore of the function layer is bigger and the rejection molecular weight is higher. The molecular weight of BSA is 67 thousand, and when a few openings with several microns appear on the surface of the membranes, the membrane almost has no any rejection ability to BSA liquor.

In Figure 3, when the mass fraction of the coagulation bath is among 0\%-50\%, the rejection ratio of PES membrane to BSA is in 60\%-70\%, without large changes. But when the DMAc concentration exceeds 50\%, the rejection ratio of PES membrane to BSA descends rapidly, and the membrane formed in the coagulation bath with $70 \%$ of DMAc, the rejection ratio has descended to about $10 \%$.

The change of the support layer has little effect on the rejection, so when the mass fraction of the first coagulation bath is $0 \%-50 \%$, the rejection ratio of the membrane to BSA changes little. In the transiting process from finger pore to the spongy pore, the flux would descend, but because the function layer has no obvious changes, so the rejection ration would be maintained in a certain range. When the DMAc content in the coagulation bath exceeds $50 \%$, the bug, that is the opening structure appears in the function layer, the rejection ratio of the function layer to BSA begins to descend, and the rejection ratio will descend rapidly with the increase of openings. When the DMAc content of the first coagulation bath achieves to $70 \%$, the membrane surface is full of disperse big pore structure which can freely pass BSA molecules, and except for the adsorption of membrane itself to the BSA molecules, the membrane has no rejection ability to BSA molecules.

\section{Conclusions}

(1) Through extending the stay time of the membrane, the surface and interior of the membrane can be implemented pretreatment and accordingly the interior and exterior structures can be changed.

(2) When the DMAc mass fraction in the first coagulation increases, the interior structure of the membrane begins to transit form the finger pore to the spongy pore, and the spongy pores increase and the finger pores become smaller and less.

(3) When the DMAc content in the first coagulation bath exceeds 50\%, the opening structure appears on the membrane surface and increases with the increase of the DMAc mass fraction.

(4) When the DMAc content in the first coagulation bath is under $50 \%$, the flux of the membrane descends and the rejection ratio change little. But when DMAc concentration exceeds $50 \%$, because the openings appear on the surface, the pure water flux rapidly ascends, and the rejection ratio to BSA descends rapidly.

\section{References}

He T, Mulder M H \& Wessling M. (2003). Preparation of porous hollow fiber membranes with a triple-orifice spinneret. Journal of Applied Polymer Science. 87(13). p.2151-2157.

Lin, Yizheng \& Smoter. (1991). Modeling on dissymmetry hollow fiber gas separation membrane by dual coagulating bath 
method. Membrane Science and Technology. 11(1).

Li S -, Koops G H \& Mulder M H, et al. (1994). Wet spinning of integrally skinned hollow fiber membranes by a modified dualbath coagulation method using a triple orifice spinneret. Journal of Membrane Science. No.94. p.329-340.

Sun, Junfen \& Wang, Qingrui. (2003). Influence of mass fraction in coagulating bath on microstructure and performance of PES ultrafiltration membrane. Journal of Donghua University (Natural Science). 29(1). p.102-105.

Wei, Yongming \& Xu, Zhenliang. (2005). Effect of the first coagulant in the double-coagulant system on the membrane structure of PSF hollow fiber membrane. Journal of Functional Polymers. 18(2).

Zhao, Youzhong, Zhu, Sijun \& Meiyong. (2005). Effect of the Second Gelation Condition on the Structures and Properties of PES Membrane. Journal of Donghua University (Natural Science). 31(5). p.93-96.

Zhu, Sijun \& Wang, Qingrui. (2005). Influence of mass fraction in coagulating bath on structure and performance of PES hollow fiber membrane. Technology of Water Treatment. 31(3). p.17-19.

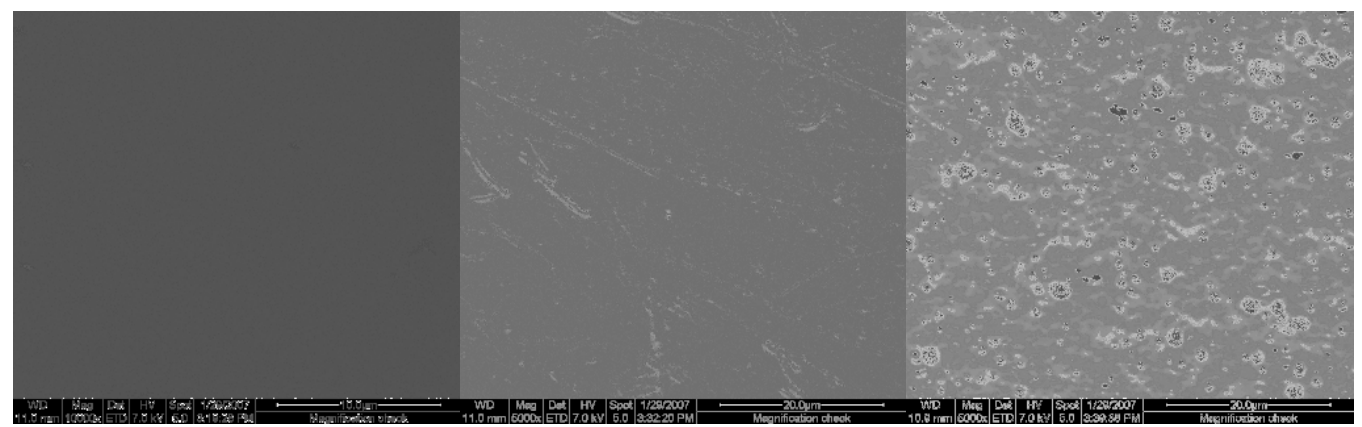

A1

$\mathrm{A} 2$

A3

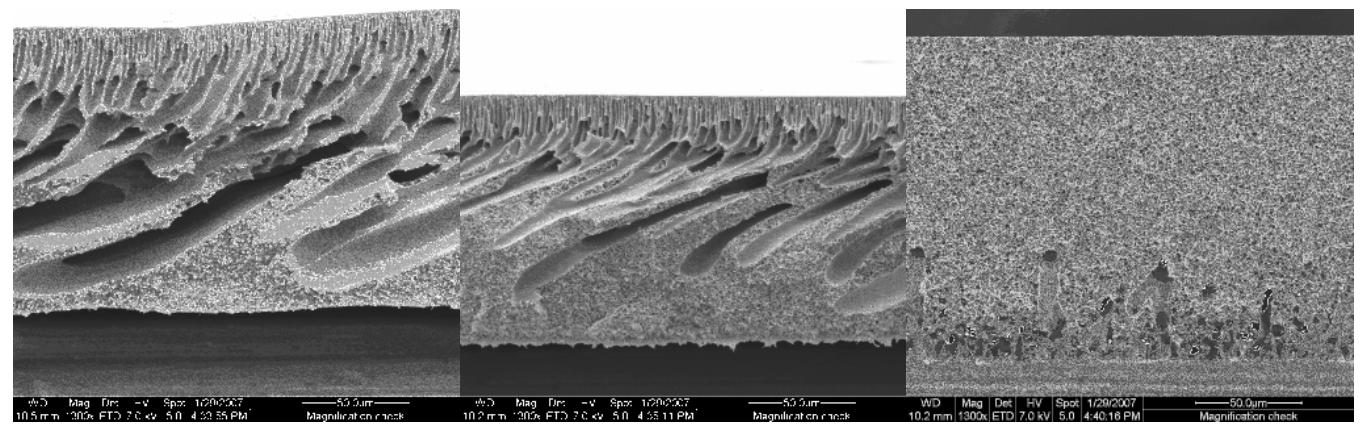

Figure 1. SEM Micrographs of External Surface (A1, A2 and A3) and Cross-section (B1,B2 and B3) Views of the Flat-sheet Type Membrane Prepared with Different DMAc Mass Fraction in Coagulation: (A1 and B1) [DMAc]=30\%, (A2 and B2) $[\mathrm{DMAc}]=50 \%$ and (A3 and B3) $[\mathrm{DMAc}]=70 \%$

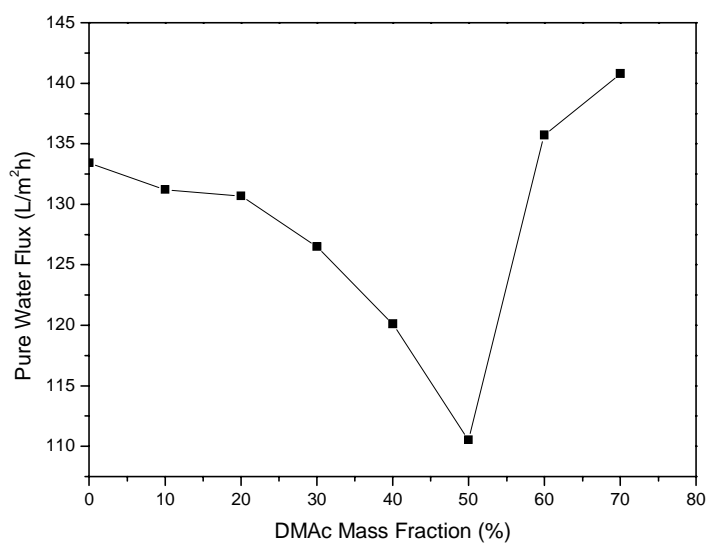

Figure 2. Effect of DMAc Mass Fraction in Coagulation on the Pure Water Flux of the Prepared Flat-sheet Type Membrane

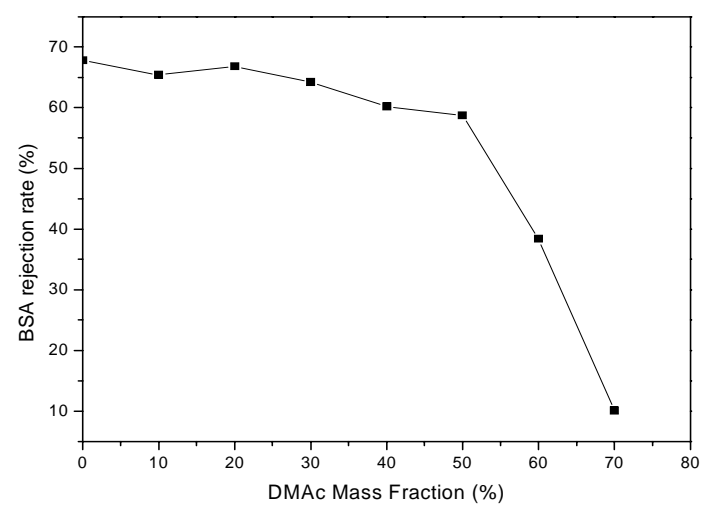

Figure 3. Effect of DMAc Mass Fraction in Coagulation on the BSA Rejection Rate of the Prepared Flat-sheet Type Membrane 